\title{
Letter to Editor about "New index for the diagnosis of liver fibrosis in Schistosomiasis mansoni"
}

\author{
Carta ao Editor sobre "Novo índice biológico para o diagnóstico \\ da fibrose hepática na Esquistossomose mansoni"
}

\author{
Ana Virgínia Matos Sá BARRETO
}

Received 18/2/2019 Accepted 27/2/2019

\section{Dear Editor of Archives of Gastroenterology,}

In the first issue of 2017 we published the paper entitled "New index for the diagnosis of liver fibrosis in Schistosomiasis mansoni"(1), which describes a new score to predict the pattern of periportal fibrosis in patients with schistosomiasis mansoni.

Recently, reassessing our dataset used in this published paper drive application in clinical practice, we detected that results presented in TABLE 2 (page 53), referring to the cut-off points separating the fibrosis groups, were incorrect.
The formula proposed to index is:

Coutinho-index $=\mathrm{AP}(/ \mathrm{ULN}) /$ platelet count $\left(\mathrm{x} 10^{9} \mathrm{cel} / \mathrm{mm}^{3}\right)$ $\mathrm{x} 100$, where $\mathrm{AP}=$ alkaline phosphatase and $\mathrm{ULN}=$ upper limit of normality.

Seeking the error, after some calculations, we found out that in the final version of published article, the serum levels of ALP "weren't" divided by ULN.

After error detection, we revised and restored all calculations dividing the serum levels of AP by ULN. The results are highlighted in red and should now be placed (TABLES 1 and 2 and FIGURE 1).

TABLE 1. Univariate analysis of serum levels of biomarkers in 116 patients with schistosomiasis mansoni.

\begin{tabular}{|c|c|c|c|c|}
\hline \multirow[b]{2}{*}{ Biomarkers } & \multicolumn{3}{|c|}{ Periportal fibrosis group } & \multirow{2}{*}{$P$ value* } \\
\hline & $\mathrm{A}+\mathrm{B}($ median $) \mathrm{n}=19$ & $\mathrm{C}+\mathrm{D}($ median $) \mathrm{n}=48$ & $\mathrm{E}+\mathrm{F}($ median $) \mathrm{n}=49$ & \\
\hline Alanine aminotransferase (/ULN) & $0.61(0.45-0.71)$ & $0.67(0.46-0.92)$ & $0.77(0.61-1.12)$ & $0.0052^{\mathrm{b}}$ \\
\hline Aspartate aminotransferase (/ULN) & $0.61(0.52-0.71)$ & $0.75(0.58-1.05)$ & $0.87(0.71-1.13)$ & $<0.001^{\mathrm{b}}$ \\
\hline$\gamma$-Glutamyl transferase (/ULN) & $0.55(0.38-0.74)$ & $0.87(0.49-1.94)$ & $1.52(0.92-3.13)$ & $<0.001^{\mathrm{b}}$ \\
\hline Hyaluronic acid (ng/ml) & $22.5(19-37.1)$ & $32.7(20.7-50.6)$ & $67.8(37.6-102.8)$ & $<0.001^{\mathrm{b} ; \mathrm{c}}$ \\
\hline TNF- $\alpha(\mathrm{pg} / \mathrm{mL})$ & $1.5(0-2.2)$ & $0.8(0-4.4)$ & $1.5(0-4.7)$ & - \\
\hline IL-13 (pg/mL) & $26.4(20.4-42.8)$ & $26.6(16.7-38.4)$ & $26.4(15-5-39-8)$ & - \\
\hline TGF- $\beta(\mathrm{pg} / \mathrm{mL})$ & $58.350(40.698-74.929)$ & $35.534(14.085-56.575)$ & $23.793(14.442-36.410)$ & $<0.001^{\mathrm{b}}$ \\
\hline
\end{tabular}

$\mathrm{A}+\mathrm{B}=$ without PPF $\mathrm{C}+\mathrm{D}=$ mild to moderate PPF $\mathrm{E}+\mathrm{F}=$ advanced PPF; ULN - upper limit of normality; *Mann-Whithey test $;$ Median $\left(\mathrm{P}_{25}-\mathrm{P}\right.$ 75 $) \cdot \mathrm{a}-\mathrm{A}+\mathrm{B} \times \mathrm{C}+\mathrm{D} ; \mathrm{b}-\mathrm{A}+\mathrm{B} \times \mathrm{E}+\mathrm{F} ; \mathrm{c}-\mathrm{C}+\mathrm{D} \times \mathrm{E}+\mathrm{F}$. 
TABLE 2. Performance of the Coutinho-index as predictors of the periportal fibrosis patterns in 116 schistosomiasis patients.

\begin{tabular}{|c|c|c|c|c|c|c|c|}
\hline & n $(\%)$ & $\mathrm{n}(\%)$ & $95 \% \mathrm{CI}$ & $95 \% \mathrm{CI}$ & $95 \% \mathrm{CI}$ & $\%$ & $\%$ \\
\hline & $A+B(n=19)$ & $\mathrm{C}+\mathrm{D}+\mathrm{E}+\mathrm{F}(\mathrm{n}=97)$ & AUC & Sensitivity & Specificity & PPV & NPV \\
\hline Coutinho-index & & & 0.907 & & & & \\
\hline$<0.33$ & $17(89.5)$ & $18(18.5)$ & & 81.4 & 94.7 & 97.5 & 48.6 \\
\hline$\geq 0.33$ & $2(10.5)$ & $79(81.4)$ & & & & & \\
\hline Coutinho-index & & & 0.819 & & & & \\
\hline \multicolumn{8}{|l|}{ Cut off } \\
\hline$<0.30$ & $17(89.5)$ & $14(29.2)$ & & 70.8 & 89.5 & 94.4 & 54.8 \\
\hline$\geq 0.30$ & $2(10.5)$ & $34(70.8)$ & & & & & \\
\hline \multirow[t]{2}{*}{$\geq 0.33$} & $2(10.5)$ & $48(98)$ & & & & & \\
\hline & $C+D(n=48)$ & $\mathrm{E}+\mathrm{F}(\mathrm{n}=49)$ & AUC & Sensitivity & Specificity & PPV & NPV \\
\hline Coutinho-index & & & 0.791 & & & & \\
\hline \multicolumn{8}{|l|}{ Cut off } \\
\hline$<0.51$ & $31(64.6)$ & $4(8.2)$ & & 91.8 & 64.6 & 72.6 & 88.6 \\
\hline$\geq 0.51$ & $17(35.4)$ & $45(91.8)$ & & & & & \\
\hline
\end{tabular}

Coutinho-index $=\mathrm{AP}(/ \mathrm{ULN}) /$ Platelet count $\left(\mathrm{x} 10^{9} \mathrm{cel} / \mathrm{mm}^{3}\right) \mathrm{x} 100 . \mathrm{A}+\mathrm{B}=$ without PPF $\mathrm{C}+\mathrm{D}+\mathrm{E}+\mathrm{F}=$ with PPF $\mathrm{C}+\mathrm{D}=$ mild to moderate PPF; $+\mathrm{F}=$ advanced $\mathrm{PPF}$. PPV: positive predictive value; NPV: negative predictive value.

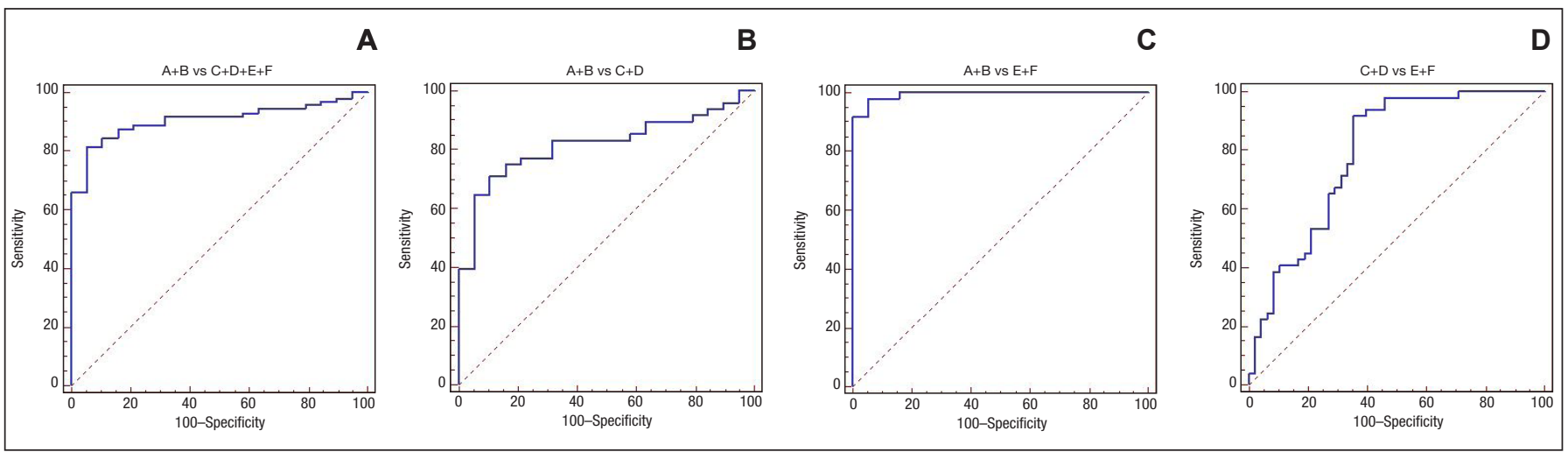

FIGURE 1. Coutinho-index ROC curves for groups of periportal fibrosis in Schistosomiasis mansoni patients. A) A+B x C+D+E+F. AUC=0.907. B) A+B x C+D. AUC=0.819. C) A+B x E+F. $A U C=0.994$. D) $C+D \times E+F$. AUC $=0.791 . A+B=$ without $P P F ; C+D+E+F=$ with $P P F ; C+D=$ mild to moderate $P P F ; E+F=$ advanced $P P F$.

Despite the alteration of the alkaline phosphatase averages/ ULN observed in TABLE 1, there were no substantial changes in ROC curves and the sensitivity and specificity between the groups studied (TABLE 2).
We apologize to the Editorial Board of the Archives of Gastroenterology for any inconvenience caused to the Journal or its readers, but we feel forced to repair our failure.

\section{REFERENCE}

1. Barreto AVMS, Alecrim VM, Medeiros TB, Domingues ALC, Lopes EP, Martins JRM, Nader HB, Diniz GTN, Montenegro SML, Morais CNL. New index for the diagnosis of liver fibrosis in Schistosomiasis mansoni. Arq Gastroenterol. 2017,54: 51-6. 